\title{
El hombro doloroso a través del razonamiento clínico
}

\author{
M. A. Udaondo Cascante, G. de Teresa Romero, V. Casado Vicente ${ }^{1}$ \\ Residente en Medicina Familiar y Comunitafispecialista en Medicina Familiar y \\ Comunitaria. Centro de Salud Parquesol. Valladolid
}

\section{RESUMEN}

El hombro doloroso es una patología muy fre cuente del aparato locomotor. El factor tiempo y los niveles de incertidumbre de nuestra consulta, hacen imprescindible seguir un esquema etiopato génico claro que incluye múltiples patologías, unas, frecuentes, a tener siempre presentes en nuestro razonamiento clínico, y otras que, por su menor frecuencia, no siempre forman parte de la primera hipótesis diagnóstica, pero que no deben olvidarse cuando las primeras no explican el cua dro clínico.

Nuestro objetivo es presentar un caso de hombro doloroso poco prevalente, aplicando el método de razonamiento clínico para su diagnóstico y trata miento.

Palabras clave: Hombro doloroso. Neuralgia amiotrófica. Síndrome de Parsonage-Turner.
The painful shoulder based on clinical decision making

\begin{abstract}
Painful shoulder is one of the commonest con sultations in Primary Health Care. Due to the lack of time and the high degree of uncertainty in our daily work, it is essential to keep in mind a clear enough etiopathogenic algorithm for a right clini cal decision making. This algorithm would include a great diversity of pathologies, some frequent enough to be always considered in our clinical rea soning, and others, less frequent, that should be considered when the first do not rationally explain the situation. The objective is not only to describe the different etiologies of the painful shoulder, but also to present this pathology with its clinical diffe rential diagnosis and treatment schema in Primary Health Care.
\end{abstract}

Key words: Painful shoulder. Amiotrophic neu ralgia. Parsonage-Turner syndrome.

\section{INTRODUCCIÓN}

El hombro doloroso es una de las patologías del aparato locomotor más frecuentes en Atención Primaria. Por el compromiso temporal y el grado de incertidumbre que caracteriza nuestra consulta diaria, es imprescindible tener en mente un esquema etiopatogénico diferencial claro si queremos realizar un abordaje correcto. Este esquema incluye una gran variedad de patologías, unas lo suficientemente frecuentes como para tenerlas siempre presentes en nuestro razonamiento clínico, y otras que, por su menor prevalencia, no siempre forman parte del primer diagnóstico diferencial, pero que no deben olvidarse cuando las primeras no explican coherentemente el cuadro clínico.

Seguidamente vamos a presentar un caso de hombro doloroso con el correspondiente esquema 
de razonamiento clínico para llegar a su diagnóstico y tratamiento, no incidiendo únicamente en una de las múltiples causas de esta entidad nosológica, sino comentando los pasos a seguir ante la misma en el contexto de la Atención Primaria.

\section{CASO CLÍNICO}

Varón de 44 años de edad, sin antecedentes familiares, personales ni laborales de interés, salvo ser fumador de 40 cigarrillos al día y bebedor moderado (16 gramos de alcohol por semana) que acudió al Servicio hospitalario de Urgencias por presentar dolor en región escapular izquierda de inicio brusco, irradiado a hombro ipsilateral, sin antecedente traumático alguno, y que se incrementaba por la noche, llegando en ocasiones a despertarle. En el Servicio de Urgencias se objetiva limitación de la movilidad, especialmente de la abducción activa, con radiografía antero-posterior y oblicua de hombro normales, radiografía de tórax normal y electrocardiograma sin alteraciones y hemograma y perfil de reconocimiento (glucosa, urea, creatinina, GOT, GPT, LDH, CK, CK-MB) normales, siendo dado de alta con el diagnóstico de dorsobraquialgia e iniciándose tratamiento con antiinflamatorios no esteroideos (AINEs) por vía oral.

Una vez en nuestra consulta se objetiva en la $e x$ ploración física postura antiálgica (brazo en extensión y addución), sin asimetrías, deformaciones ni atrofias evidentes. A la palpación, presenta dolor en cara lateral de hombro, por debajo de la articulación acromio-clavicular y a la palpación de fibras descendentes de trapecio y deltoides izquierdos, sin signos de infección (locales ni sistémicos) objetivables. Presenta, a su vez, limitación dolorosa de la abducción activa a $100^{\circ}$, de la rotación externa activa en abducción a $70^{\circ}$, de la abducción pasiva a $150^{\circ}$, y de la rotación externa pasiva en abducción a $60^{\circ}$, estando el resto de los movimientos relativamente conservados, incluida la rotación interna. Las pruebas de aprehensión fueron negativas. La sensibilidad y los reflejos osteotendinosos (ROT) fueron en todo momento normales. No existía dolor a la palpación de articulación acromioclavicular ni coracoclavicular. En la auscultación cardiopulmonar y la exploración abdominal no se encontraron hallazgos de interés. La exploración cervicodorsal fue normal.

\section{HIPÓTESIS DIAGNÓSTICAS. FASES DEL RAZONAMIENTO CLÍNICO}

\section{Fase de presentación y primera hipótesis diagnóstica}

La anamnesis y exploración física evidencian un patrón mixto, articular (limitación tanto de la mo- vilidad pasiva como de la activa) y periarticular (limitación mayor de la movilidad activa, especialmente de la abducción -ABD- y rotación externa -RE-), estableciéndose como primera hipótesis diagnóstica una tendinopatía del manguito de los rotadores, especialmente del supraespinoso, con posible componente de bursitis subacromial asociado. Por ello, se inicia tratamiento con AINEs vía oral, medidas físicas (reposo relativo, crioterapia en las primeras 72 horas, y calor después) y se remite al paciente a fisioterapia (FST).

Una semana después, el paciente refiere desapa rición del dolor, aunque empeoramiento importan te de su movilidad, especialmente de la ABD activa y pasiva, con cierta limitación de la RE, estando el resto de movimientos conservados. El resto de exploración física, incluida la neurológica, no mostró hallazgos de interés, manteniéndose la misma hipó tesis, sin variar el tratamiento.

A las dos semanas se evidencia una disminución de la fuerza (ABD: 3/5, RE: 3/5, resto: 4-5/5) con importante fatigabilidad en todos los movimientos del hombro. Se objetiva discreta asimetría de hombros con dudoso descenso de hombro izquierdo, posiblemente en relación con atrofia muscular. La sensibilidad y los ROT siguen siendo normales, así como la exploración de la columna cervical y sistémica.

\section{Fase de diagnóstico diferencial}

En la tabla I se recogen los posibles diagnósticos diferenciales del hombro doloroso, con su correspondiente clasificación del tipo de hipótesis (hipó tesis activas: aquéllas cuyo contexto clínico y epidemiológico responde inicialmente a los síntomas y signos presentados por el paciente, hipótesis inactivas: aquéllas que no se rechazan, pero la probabilidad de que el paciente presente esa enfermedad, ya sea por el contexto clínico o epidemiológico, es baja e hipótesis pasivas: aquéllas que se rechazan porque la probabilidad de que el paciente las padezca es casi inexistente por el contexto clínico o epidemiológico). En este momento, el inicio nocturno de dolor escapular, la pérdida de movilidad activa y la desaparición del dolor con empeo ramiento funcional asociado a amiotrofia incipiente van a favorecer más de un predominio de patrón periarticular o referido, haciéndonos sospechar como principales hipótesis activas la existencia de un proceso neurológico o de una tendinopatía en desarrollo (rotura parcial o total del manguito de los rotadores, si bien la atrofia es de aparición demasiado temprana), por lo que se remite al paciente al Servicio de Traumatología, a la vez que se intenta adelantar el tratamiento FST, manteniendo mientras tanto el mismo tratamiento conservador. 
Tabla I

PRINCIPALES CAUSAS DE HOMBRO DOLOROSO ${ }^{13}$

Patrón periarticular

1. Patología tendinosa ${ }^{1}$ (tendinitis vs rotura):

$M R$ : SE, IE, RM, Rm, sE

Bíceps braquial

Deltoides

2. Patología bolsas sinoviales1 (bursitis): Subacromial

3. Patología ósea:

Síndrome acromioclavicular

Síndrome coracoclavicular
Patrón articular (glenohumeral)

1. Capsulitis retráctil ${ }^{1}$ (hombro congelado)

2. Artritis ${ }^{3}$ :

Inflamatorias: AR, EA, conectivopatías

Microcristales: gota, condrocalcinosis

Infecciosas

3. Artrosis $^{2}$

4. Inestabilidad glenohumeral ${ }^{3}$

5. Necrosis ósea avascular ${ }^{3}$

6. Enfermedad de Paget $^{3}$

7. Neoplasias, metástasis, Sd. paraneoplásicos²

Patrón referido

1. Origen neurológico:

Espondiloartrosis cervical ${ }^{1}$

Subluxación cervical ${ }^{2}$

Enfermedad del plexo braquial ${ }^{1}$

Neuropatía por atrapamiento

Tumores medulares ${ }^{2}$

Parálisis del nervio torácico largo ${ }^{1}$

Neuralgia amiotrófica (Sd. Parsonage-Turner) ${ }^{1}$

Esclerosis lateral amiotrófica ${ }^{2}$

2. Origen neurovascular:

Distrofia simpático refleja (Sd. Hombro-mano) ${ }^{3}$

$\mathrm{Sd}$. del desfiladero torácico ${ }^{3}$
$\mathrm{IAM}^{3}$

Disección aorta ${ }^{3}$

TEP $^{3}$

Absceso subfrénico ${ }^{3}$

Neumotórax ${ }^{3}$

Pericarditis-pleuritis ${ }^{3}$

Mesotelioma ${ }^{3}$

Tumor Pancoast ${ }^{3}$

Infarto esplénico ${ }^{3}$

Patología vesicular ${ }^{3}$

Rotura de víscera abdominal ${ }^{3}$
3. Origen viscerosomático:

Pancreatitis-ulcus péptico ${ }^{3}$

Otras causas de hombro doloroso

Diabetes mellitus ${ }^{3}$

Hiper/ hipotiroidismo ${ }^{3}$

Hiperparatiroidismo ${ }^{3}$

Osteodistrofia renal ${ }^{3}$

MR: Manguito de los rotadores; SE: Supraespinoso; IE: Infraespinoso; RM: Redondo Mayor; Rm: Redondo menor; sE: Subescapular; AR: Artritis reumatoide; EA: Espondiloartropatías; Sd: Síndrome; IAM: Infarto agudo de miocardio; TEP: Tromboembolismo pulmonar.

${ }^{1}$ Hipótesis activas; ${ }^{2 H i p o ́ t e s i s ~ i n a c t i v a s ; ~}{ }^{3 H i p o ́ t e s i s ~ p a s i v a s ~}$

Una vez visto en el Servicio de Traumatología, se objetiva atrofia muscular de supra e infraespinoso con imposibilidad para la antepulsión y ABD y $\mathrm{RE}$ activa resistida y compromiso importante de la activa no resistida del hombro izquierdo. La exploración del subescapular y la exploración cervical es normal, así como el hombro contralateral. Ante la sospecha de rotura del manguito de los rotadores de hombro izquierdo, el traumatólogo solicita resonancia magnética nuclear (RM) y proyecciones an tero-posterior y axial de hombro.

\section{Fase de diagnóstico}

La radiografía es normal. El resultado de la $\mathrm{RM}$, pasado un mes, muestra "engrosamiento del com plejo tendinoso-rotador que afecta a todos sus componentes, con rotura intrasustancial del tendón del supraespinoso (SE) y descenso de la cabeza hu meral con buena congruencia articular glenohu meral y buen aspecto del tendón del bíceps bra quial". Dicho resultado aporta cierta información, pero no explica coherentemente el cuadro clínico, por lo que no puede considerarse el caso cerrado.

\section{Fase de explicación}

Ante la persistencia de la atrofia del SE e infraespinoso un mes más tarde, con espina escapular protruyente, aunque sin evidenciarse escápula alada y limitación de $25^{\circ}$ de la RE, se sospecha paresia de los $\mathrm{RE}$ y/o rotura de tendón del $\mathrm{SE}$, por lo que se deriva al Servicio de Rehabilitación (RHB) y se solicita electromiograma (EMG) supraescapu- 
lar, la cual muestra "en reposo actividad espontá nea en forma de fibrilación y ondas lentas en los músculos supra e infraespinoso, detectándose elec tromiográficamente alteraciones nerviosas perifé ricas en territorios de nervio circunflejo y supraes capular izquierdos en diferentes estadios de afectación, teniendo como única conexión común la raíz C5. En el contexto clínico en que se dan di chos hallazgos, todo parece indicar que se trata de un síndrome de Parsonage-Turner".

El inicio precoz de la FST y las propias características del referido síndrome hicieron que el paciente se encontrara asintomático y con normofunción de la articulación del hombro, cuatro meses después del inicio del cuadro.

\section{DISCUSIÓN}

De forma general, con una rápida anamnesis y una buena exploración física puede clasificarse el hombro doloroso en tres distintos patrones: patrón periarticular, patrón articular o patrón referido. En la práctica diaria es frecuente, no obstante, la existencia de más de uno de estos patrones simultáneamente, si bien, su distinción se basa en los mismos signos y síntomas.

El patrón periarticular se caracteriza principalmente por la mayor afectación de la movilidad acti $v a \mathrm{y}$, sobre todo, de la activa contra resistencia en los movimientos en los que participen los músculos afectados, permaneciendo la movilidad pasiva sin alteraciones. El compromiso funcional y la localización del dolor son inicialmente selectivos, para ir afectándose prácticamente todos los movimientos y la amplitud del dolor a medida que evoluciona la patología.

El patrón articular o capsular se caracteriza por la afectación sobre todo de la movilidad pasiva, especialmente en grados máximos, con o sin presencia de crepitación ósea durante la misma, pudiendo estar la movilidad activa y activa contra resistencia afectada o no. A medida que evoluciona la patología, lo habitual es que los tres tipos de movilidad se vean afectados.

El patrón referido variará según la etiología del mismo (neurológica, neurovascular o viscerosomática). Sospecharemos este patrón en función de los antecedentes personales del paciente y/o tras descartar clínicamente los patrones anteriores.

La neuralgia amiotrófica o síndrome de Parso nage-Turner es una patología descrita por primera vez por Parsonage y Turner en $1948^{1}$, también denominada neuropatía del plexo braquial, plexitis braquial aguda, neuritis aguda del hombro o neuritis paralítica, que afecta más frecuentemente a varones entre los 30 y 60 años, habiéndose descrito una relación hombre:mujer tan dispar como
11,5:12 ó $2: 1^{3}$ y cursa característicamente con intenso dolor en el hombro que va desapareciendo espontáneamente a la vez que se va instaurando una importante atrofia e impotencia funcional de la articulación; generalmente se trata de un proceso autolimitado en un tiempo variable, pasando desde meses a años hasta que se instaura la normalidad funcional. Se afectan casi siempre las segundas motoneuronas de las ramas superiores del plexo braquial, generalmente de forma múltiple (más de dos ramas), si bien no son frecuentes las fasciculaciones ${ }^{4}$, afectándose motoramente, en orden de frecuencia, el deltoides, supraespinoso, infraespinoso, bíceps, tríceps y serrato anterior, y, en menor frecuencia, los flexores y extensores de la muñeca y musculatura lumbar ${ }^{5} \mathrm{o}$, desde el punto de vista neurológico, el nervio torácico largo o el nervio supraescapular, según las series recogidas. De etiología desconocida, aunque se ha relacionado con intervenciones quirúrgicas ${ }^{1}$, factores inmunológicos, infecciones ${ }^{6,7}$, vacunas de la hepatitis $\mathrm{B}^{8} \mathrm{o}$ antigripal ${ }^{9}$, causas mecánicas ${ }^{10}$, o radioterapia asociada a linfoma de Hodgkin. Algunos autores han descrito afectación bilateral hasta en un tercio de los $\operatorname{casos}^{3}$. La afectación sensorial no es infrecuente, variando su incidencia según la serie escogida; en una de las series más largas publicadas, los autores encontraron hasta un $68 \%$ de afectación sensorial $^{3}$.

Como hemos dicho anteriormente, el inicio agudo del dolor intenso sin antecedente traumático previo, generalmente más intenso nocturno, con exploración cervical normal y la desaparición del dolor a medida que se inicia la impotencia funcional y la atrofia, nos orienta hacia el diagnóstico. No obstante, se trata de un diagnóstico de exclusión.

La prueba "gold standard" para diagnosticar esta patología es la electromiografía, que localizará, a su vez, el lugar exacto de la lesión. La RM mostrará un aumento en la intensidad de la señal de los músculos afectados en $\mathrm{T}_{2}$, así como una mayor o menor atrofia muscular en función del momento de su realización. $\mathrm{Su}$ valor reside en la información que nos aporta para descartar otras patologías (radiculopatías, tumores medulares, etc.).

El pronóstico es habitualmente muy bueno, siendo autolimitada y con recuperación absoluta en un tiempo variable con tratamiento puramente sintomático. Helms et al $^{11}$ describieron tres casos con recuperación en un tiempo de dos a cuatro meses. Dillin et al ${ }^{12}$ describieron una recuperación funcional casi completa también en los primeros meses de evolución, mientras que Magee and De Jong ${ }^{2}$ encontraron que la recuperación completa podía prolongarse hasta 8 años, estableciendo una asociación entre el tiempo de duración del dolor y el de recuperación funcional. Como tratamiento sintomático se ha probado la fisioterapia precoz, los AINEs 
orales o intramusculares, los analgésicos de potencia leve y media y los corticoides orales, parenterales o intraarticuares, no habiendo sido establecidos aún los beneficios de éstos en relación con los dos primeros ${ }^{4}$. Ningún protocolo terapéutico contempla la cirugía entre sus posibilidades.

\section{AGRADECIMIENTO}

A Ramón Herreros López, Especialista en Traumatología del Hospital Universitario Río Hortega de Valladolid, por su colaboración en este trabajo y por ser un excelente referente para los Médicos de Familia en el segundo nivel.

\section{CORRESPONDENCIA:}

M. A. Udaondo Cascante

Centro de Salud Parquesol

C/ Ciudad de la Habana, s/n

47014 Valladolid

\section{Bibliografía}

1. Parsonage MJ, Turner JWA. Neuralgic amyotrophy. The shoulder-girdle syndrome. Lancet 1948; 1: 973-8.

2. Magee KR, DeJong RN. Paralytic brachial neuritis. JAMA 1960; 174: 1258-62.

3. Tsairis P, Dyck PJ, Mulder DW. Natural history of brachial plexus neuropathy: report on 99 patients. Arch Neurol 1972; 27: 109-17.

4. Aymond JK, Goldner JL, Hardaker WT. Neuralgic amyotrophy. Orthop Rev 1989; 12: 1275-9.

5. Rifisch A, van Laack W. Neuralgic amyotrophy of the lumbar area. Acta Orthop Trauma Surg 1989; 108: 329-32.

6. Misamore GW, Lehman DE. Parsonage-Turner syndrome (acute brachial neuritis). J Bone Joint Surg Am 1996; 78: 1405-8.

7. Pellas F, Olivares JP, Zandotti C, Delarque A. Neuralgic amyotrophy after parvovirus B19 infection. Lancet 1993; 342: 503-4.

8. Reutens DC, Dunne JW, Eláter H. Neuralgic amyotrophy following recombinant DNA hepatitis B vaccination. Muscle Nerve 1990; 13: 461

9. Miller JD, Pruitt S, McDonald TJ. Acute brachial plexus neuritis: an uncommon cause of shoulder pain. Am Fam Phys 2000; 62: 2067-72.

10. Pou Serradell A, Moral Pijaume A. Síndrome de Parsonage y Turner. A propósito de 25 casos. Rev Neurol 1988; 78: 7-16.

11. Helms CA, Martínez S, Speer KP. Acute brachial neuritis (Parsonage-Turner syndrome): MR imaging appearancereport of three cases. Radiology 1998; 207: 255-9.

12. Dillin L, Hoaglund FT, Scheck M. Brachial neuritis. J Bone Joint Surg Am 1985; 67: 878-80. 